\title{
THE RELATION BETWEEN THE SEQUENCE-TO-SEQUENCE AND THE SERIES-TO-SERIES VERSIONS OF QUASI- HAUSDORFF SUMMABILITY METHODS
}

B. KWEE

1. Introduction. Let $\left(H, \mu_{n}\right)$ be a regular Hausdorff method of summability, and let

$$
\begin{aligned}
& t_{n}=\sum_{k=n}^{\infty}\left(\begin{array}{l}
k \\
n
\end{array}\right)\left(\Delta^{k-n} \mu_{n+1}\right) s_{k}, \\
& b_{n}=\sum_{k=n}^{\infty}\left(\begin{array}{l}
k \\
n
\end{array}\right)\left(\Delta^{k-n} \mu_{n}\right) a_{k},
\end{aligned}
$$

where $s_{k}=a_{0}+a_{1}+\cdots+a_{k}$. We shall call $A$ the summability method given by the sequence-to-sequence transformation (1), and $B$ the summability method given by the series-to-series transformation (2). It is proved in [2] and [3] that summabilities $A$ and $B$ are regular.

We shall say that the transformations (1) and (2) are equivalent if the convergence of (1) for all $n$ implies the convergence of (2) for all $n$, and conversely, and in either case, the sums are related by the equation

$$
t_{n}=b_{0}+b_{1}+\cdots+b_{n} .
$$

(1) may be written as

$$
t=H^{*}\left(\mu_{n+1}\right) s
$$

where $s, t$ denote the sequences $\left(s_{k}\right),\left(t_{k}\right)$, and $H^{*}\left(\mu_{n+1}\right)$ the matrix $\left(\alpha_{n, k}\right)$, where

$$
\begin{aligned}
\alpha_{n, k} & =\left(\begin{array}{l}
k \\
n
\end{array}\right)\left(\Delta^{k-n} \mu_{n+1}\right) \quad(k \geqq n), \\
& =0 \quad(k<n) .
\end{aligned}
$$

We shall prove the following two theorems.

THEOREM 1. If $t_{0}=b_{0}$, and

$$
H^{*}\left(\mu_{n+1}\right)\left\{H^{*}(n+1) s\right\}=H^{*}(n+1)\left\{H^{*}\left(\mu_{n+1}\right) s\right\},
$$

then the transformations (1) and (2) are equivalent.

Theorem 2. If, for all (fixed) $n$,

Received by the editors May 18, 1964 and, in revised form, November 21, 1966. 


$$
\left(\begin{array}{l}
k \\
n
\end{array}\right)\left(\Delta^{k-n} \mu_{n}\right) s_{k-1} \rightarrow 0
$$

as $k \rightarrow \infty$, then the transformations (1) and (2) are equivalent.

2. Proof of Theorem 1. Let $\bar{a}$ and $\bar{b}$ denote the sequences $\left\{(n+1) a_{n+1}\right\}$ and $\left\{(n+1)\left(t_{n+1}-t_{n}\right)\right\}$. Then $\bar{a}=-H^{*}(n+1) s$, and, by $(4)$,

$$
\begin{aligned}
\bar{b} & =-H^{*}(n+1) t=-H^{*}(n+1)\left\{H^{*}\left(\mu_{n+1}\right) s\right\} \\
& =-H^{*}\left(\mu_{n+1}\right)\left\{H^{*}(n+1) s\right\} \\
& =H^{*}\left(\mu_{n+1}\right) \bar{a} .
\end{aligned}
$$

Hence

$$
(n+1)\left(t_{n+1}-t_{n}\right)=\sum_{k=n}^{\infty}\left(\begin{array}{l}
k \\
n
\end{array}\right)\left(\Delta^{k-n} \mu_{n+1}\right)(k+1) a_{k+1}
$$

for $n \geqq 0$. Noting that $(k+1 / n+1) \mathrm{C}_{k, n}=\mathrm{C}_{k+1, n+1}$ and replacing $k+1$ by $k$ and $n+1$ by $n$, we have

$$
t_{n}-t_{n-1}=\sum_{k=n}^{\infty}\left(\begin{array}{l}
k \\
n
\end{array}\right)\left(\Delta^{k-n} \mu_{n}\right) a_{k}=b_{n}
$$

for $n \geqq 1$, and $t_{0}=b_{0}$ by hypothesis. Thus (3) is satisfied, and the transformations (1) and (2) are equivalent.

3. Proof of Theorem 3. Write

$$
\begin{aligned}
b_{n, K} & =\sum_{k=n}^{K}\left(\begin{array}{l}
k \\
n
\end{array}\right)\left(\Delta^{k-n} \mu_{n}\right) a_{k}, \\
t_{n, K} & =\sum_{k=n}^{K}\left(\begin{array}{l}
k \\
n
\end{array}\right)\left(\Delta^{k-n} \mu_{n+1}\right) s_{k}
\end{aligned}
$$

(both of these may be taken as 0 for $n>K$ ). If (5) holds, then, for any fixed $n$, we have, as $K \rightarrow \infty$

$$
\begin{aligned}
b_{n, K} & =\sum_{k=n}^{K}\left(\begin{array}{l}
k \\
n
\end{array}\right)\left(\Delta^{k-n} \mu_{n}\right)\left(s_{k}-s_{k-1}\right) \\
& =\sum_{k=n-1}^{K} s_{k} \Delta\left\{\left(\begin{array}{l}
k \\
n
\end{array}\right)\left(\Delta^{k-n} \mu_{n}\right)\right\}+o(1),
\end{aligned}
$$

where the $\Delta$ outside the curly bracket is taken as operating on the variable $k$, and the curly bracket is taken as 0 when $k=n-1$. Now using 


$$
\Delta^{k-n} \mu_{n}=\Delta^{k+1-n} \mu_{n}+\Delta^{k-n} \mu_{n+1}
$$

we have

$$
\Delta\left\{\left(\begin{array}{l}
k \\
n
\end{array}\right)\left(\Delta^{k-n} \mu_{n}\right)\right\}=\left(\begin{array}{l}
k \\
n
\end{array}\right)\left[\Delta^{k+1-n} \mu_{n}+\Delta^{k-n} \mu_{n+1}\right]-\left(\begin{array}{c}
k+1 \\
n
\end{array}\right) \Delta^{k+1-n} \mu_{n}
$$

$$
=-\left(\begin{array}{c}
k \\
n-1
\end{array}\right) \Delta^{k-(n-1)} \mu_{n}+\left(\begin{array}{l}
k \\
n
\end{array}\right) \Delta^{k-n} \mu_{n+1},
$$

where we take the second term on the right of (9) as meaning 0 in the case $k=n-1$, and the first as meaning 0 when $n=0$.

We deduce at once from (8) and (9) that, for fixed $n$,

$$
t_{n, K}=b_{0, K}+b_{1, K}+\cdots+b_{n, K}+o(1)
$$

as $K \rightarrow \infty$, and this proves the theorem.

4. Examples. Now let us apply these ideas to some examples. We shall use the following lemma which is a paraphrase of Theorem 26 in $[1]$.

Lemma. If, for any sequence $\left(p_{k}\right)$ which is monotonic decreasing for large enough $k, \sum_{k=n}^{\infty} a_{k} p_{k}$ exists, then

$$
\lim _{k \rightarrow \infty} p_{k} \sum_{l=n}^{k} a_{l}=0
$$

(i) If $\mu_{n}=\lambda^{n}(0<\lambda<1)$, then (1) becomes

$$
t_{n}=\lambda^{n+1} \sum_{k=n}^{\infty}\left(\begin{array}{l}
k \\
n
\end{array}\right)(1-\lambda)^{k-n} s_{k} .
$$

This is the circle method of summation introduced by Hardy and Littlewood. (2) becomes

$$
b_{n}=\lambda^{n} \sum_{k=n}^{\infty}\left(\begin{array}{l}
k \\
n
\end{array}\right)(1-\lambda)^{k-n} a_{k},
$$

and (5) becomes

$$
\left(\begin{array}{l}
k \\
n
\end{array}\right)(1-\lambda)^{k-n} s_{k-1} \rightarrow 0
$$

The convergence of (10) for a given $n$ implies (12) for that $n$. Also, by the lemma quoted above with $p_{k}=\mathrm{C}_{k, n}(1-\lambda)^{k-n}$, the convergence of (11) for a given $n$ implies (12). 
Since summability $A$ asserts more than the convergence of (10) for all $n$, and summability $B$ asserts more than the convergence of (11) for each $n$, we see at once that, in this case, summabilities $A$ and $B$ are equivalent.

(ii) If

$$
\mu_{n}=\left(\begin{array}{c}
n+r \\
r
\end{array}\right)^{-1}
$$

then (1) becomes

$$
\begin{aligned}
t_{n} & =r(n+1) \sum_{k=n}^{\infty} \frac{k(k-1)(k-2) \cdots(k-n+1)}{(k+r+1)(k+r) \cdots(k+r-n)} s_{k} \\
& =\frac{n+1}{r+1} \Delta^{-r}\left(\frac{s_{n}}{\left(\begin{array}{c}
n+r+1 \\
n
\end{array}\right)}\right) .
\end{aligned}
$$

This is the quasi-Cesáro transformation $\left(C^{*}, r\right)$ introduced by Kuttner [4]. (2) becomes

$$
\begin{aligned}
b_{n} & =r \sum_{k=n}^{\infty} \frac{k(k-1) \cdots(k-n+1)}{(k+r)(k+r-1) \cdots(k+r-n)} a_{k} \\
& =\Delta^{-r}\left\{\frac{a_{n}}{\left(\begin{array}{c}
n+r \\
n
\end{array}\right)}\right\} .
\end{aligned}
$$

For any given $n$, the assertion that the series defining $t_{n}$ converges is easily seen to be equivalent to

$$
\sum_{k=1}^{\infty} \frac{s_{k}}{k^{2}}
$$

converges, while the assertion that the series defining $b_{n}$ converges is equivalent to

$$
\sum_{k=1}^{\infty} \frac{a_{k}}{k}
$$

converges. Condition (5) is easily seen to reduce, in the special case considered, to

$$
s_{k}=o(k) \text {. }
$$

By the lemma quoted above with $p_{k}=1 / k,(16)$ implies (17). Hence, whatever $r, B \Rightarrow A$. On the other hand, it is clearly false that (15) 
implies (17). But summability $A$ asserts more than the convergence of (15), since (15) merely gives the existence of $t_{n}$. Thus this does not exclude the possibility that summability $A$ might imply (17).

What we do, in fact, have is that $A \Rightarrow B$ is true when, $r \leqq 1$, but not when $r>1$. For recall that $A$ is $\left(C^{*}, r\right)$. It follows from the results of a paper by Kuttner [4] that $\left(C^{*}, r\right) \Rightarrow(C, 1)$ when $r \leqq 1$; and it is well known that $(C, 1)$ implies (17). On the other hand, if $r>1$, let $1 \leqq \beta<\alpha<r$, and $s_{k}=(-1)^{k}(k+1)^{\beta}$. Then $\left(s_{k}\right)$ is summable $(C, \alpha)$, and hence summable $\left(C^{*}, r\right)[4]$. But (17) is false. Indeed, (16) does not converge, so that $b_{n}$ is not defined.

\section{REFERENCES}

1. G. H. Hardy, Divergent series, Clarendon Press, Oxford, 1949.

2. M. S. Ramanujan, On Hausdorff and quasi-Hausdorff methods of summability, Quart. J. of Math. Oxford Ser. (2) 8 (1957), 197-213.

3. - Series-to-series quasi-Hausdorf transformation, J. Indian Math. Soc. 17 (1953), 47-53.

4. B. Kuttner, On quasi-Cesaro summability, J. Indian Math. Soc. 24 (1960), 319-341.

University of Malaya, Kuala Lumpur, Malaysia 